tinuous fast wave activity, which appeared immediately after the loss of consciousness, and at first its frequency and amplitude were $20-24 \mathrm{cps}$ and 30-40 $\mu \mathrm{V}$ in lightest anesthestetic level, than it increased in voltage, and decreased in frequency. As the anesthetic level was deepend, however, slow wave activity did not appear before the overdosage stage of Fluothane anesthesia.

2) When nitrous oxide was added in inhaled gas mixture at the rate higher than $50 \% \mathrm{v} / \mathrm{v}$. E.E.G. represented slow wave activity in the lighter anesthesia level, i.e. as soon as the patient became unconscious.

3) Transition in E.E.G. pattern from Fluothane to cyclopropane and vise versa were observed when Flothane was replaced with cyclopropane in inhaled gas mixture and vice versa.

4) E.E.G. in Penthrane $\left(\mathrm{CH}_{3} \mathrm{OCF}_{2} \mathrm{CHCl}_{2}\right)$ anesthesia also revealed predominat fast wave activity similar to that in Fluothane-oxygen anesthesia.

5) In Chloroform anesthesia, E.E.G. pattern was likened to that of ether or cyclopropane in which slow wave was predominant as the anesthetic level become deeper.

From the results thus obtained, it is suggested by authors that fast wave activity would be essential characteristics in E.E.G. pattern of the anesthesia with fluorinated organic compounds.

\title{
A7. The Changes of Meninges in Cervical Syndrome.
}

\author{
T. IWahara and M. Hosokawa
}

Dept. of Ortho. Keio Univ. Hospital

Pathological changes of midpost portion of dural canal presenting cervical syndrome by cervical deformans have been studied.

Operations revealed the facts that there are adhesive and cicatric degenerations in the epidural tissue, and also thickening, adhesion and turbidness in the dura and arachnoidea.

Pathological findings showed as followings. Congestion, appearance of fibrine and infiltration of leucocytes are seen in the epidural tissue. Increased connective tissue, irregularity of fibrous structure, lack of cellular compornent and condensed nuclei are observed in the dura. Elastic fibers are variable in size, torn off in shape and disappeared at the portion of necrotic and hyalinageous degeneration.

Around the necrotic foci, there is no conspicuous histopathological reaction. Blood vessels are thickened. From time to time, there is a picture of lime clinging to the dura.

Those changes above mentioned, may be caused by gradual compression of osteophyte due to cervical deformans toward the dural canal; and also by the 
yearly change of dura itself, or circulatory disturbance.

On the other hand we can not miss the effect that the dura which already developed pathological changes, has influence to some extend to the progress of spinal complaint.

\title{
A8. Radiological Studies of Cervical Disc Syndrome.
}

\author{
M. TSURU, T. TAKEDA and K. YADA
}

The radiological appearances of twenty-five cases of cervical disc syndrome, all confirmed by surgical operation, were analyzed. There were eight cases of disc hernias and seventeen spondylosis.

While the plain X-ray demonstrated narrowing of intervertebral space, posterior osteophytosis, or foraminal narrowing, or various combination of these in the most of the cases of cervical spondylosis, these abnormalities were rarely found among the disc hernias. The posterior osteophytosis and the foraminal narrowing were often noticed in multiple disc spaces in spondylosis.

The myelogram showed either complete block or large filling defect in most of the disc cases and anterior defect or deformity of root sleeves in the cases of spondylosis. There were several cases which demonstrated marked anterior defects by myelogram but failed to find posterior osteophytosis by plaim X-ray.

Although the cervical spondylosis and the cervical disc hernias are considered to be the same group of diseases, there are significant differences in their radiological appearances. Also we would like to point out that plain X-ray often fails to demonstrate this type of pathological processes.

\section{A9. Arnold-Chiari Malformation Associated with Spina Bifida.}

\section{S. Shirakawa, S. Ono and H. Nomura}

The 2nd Surg., Iwate Med. College

Three cases (females of 6, 10 years old and 2 months) of Arnold-Chiari malformation associated with spina bifida, platybasia and hydrocephalus were reported. One of them was combined with Dandy-Walker's syndrome.

Two of three cases were cured by suboccipital decompression. It is considered that all these malformations were caused by the traction of spinal cord with spina bifida.

The basal angle of 618 cases of various kinds of diseases were measured in X-ray photography of skull in order to investigate the relation between ArnoldChiari malformation and platybasia. 